\title{
Stability of Bounded Solutions of Linear Functional Equations
}

\author{
By Joel N. Franklin
}

\begin{abstract}
The weak sequential compactness of reflexive Banach spaces is used to explain the fact that certain ill-posed, linear problems become well-posed if the solutions are required to satisfy a prescribed bound. Applications are made to the computability of solutions of ill-posed problems associated with elliptic and parabolic partial differential equations.
\end{abstract}

1. Introduction. In defining the concept of a well-posed problem, J. Hadamard [1] recognized that the existence and the uniqueness of the solution are not enough to ensure the computability of the solution. Numerical computation requires that the solution have stability, or continuous dependence on the data.

F. John [2], [3], [4] and other authors have shown that, for certain problems whose solutions have existence and uniqueness but not stability, if the solutions considered are required to satisfy a prescribed bound, stability results. An extensive bibliography appears in the paper [5] by L. E. Payne. All these results depend on precise definitions of "continuous dependence on data" which are suitable for the particular problems discussed. Much of the work preceding the present paper contains not merely qualitative statements of stability, but quantitative inequalities satisfied in particular problems.

The present paper contains only the qualitative observation that an elementary result, Theorem 1, on the weak topology of reflexive Banach spaces yields the stability of uniformly bounded solutions of a large class of linear functional equations. Several new applications are made, including a theorem on the stability of solutions of the final-value problem corresponding to the general initial-value problem discussed in the book [6] by R. D. Richtmyer.

2. Notation and Review. We shall use the letter $T$ to stand for a bounded linear operator mapping a Banach space $B_{1}$ into a Banach space $B_{2}$. We suppose that the range $T B_{1}$ is dense in $B_{2}$, and that $T z=0$ only if $z=0$.

If $B$ is a Banach space, the notation $B^{*}$ represents the conjugate Banach space of bounded linear functionals $f$ mapping $B$ into the real numbers. If $x \in B$ and $f \in B^{*}$, the notation $(x, f)$ represents the number which results when $f$ is applied to $x$. If $B^{* *}=B$, the space $B$ is called reflexive [7]. Hilbert spaces are reflexive Banach spaces. If $1<p<\infty$, the space $L^{p}$ of measurable functions $x(t)$ for which

$$
\int_{a}^{b}|x(t)|^{p} d t<\infty
$$

Received August 26, 1970.

AMS 1970 subject classifications. Primary 65M30, 65N99, 65R05; Secondary 65J05.

Key words and phrases. Well-posed, ill-posed, continuous dependence on data, stability, backwards heat-equation, final-value problem, elliptic continuation.

Copyright $\odot$ 1971, American Mathematical Society 
is reflexive. In fact, $\left(L^{p}\right)^{*}=L^{Q}$ if $1 / p+1 / q=1$. However, the Banach space $C$ of continuous functions under the maximum-norm is not reflexive.

If $\left\{x_{n}\right\}$ is a sequence of $B$, we say that it converges, or converges strongly, to $x$ if $\left\|x_{n}-x\right\| \rightarrow 0$ as $n \rightarrow \infty$; we then write $x_{n} \rightarrow x$. We say that $x_{n}$ converges weakly to $x$ if, for each $f$ in $B^{*},\left(x_{n}, f\right)$ tends to the limit $(x, f)$ as $n \rightarrow \infty$; we then write $x_{n} \rightarrow x$. A convergent sequence is weakly convergent, but the converse is false.

If $g$ lies in the range of $T$, the equation

$$
T u=g
$$

has a unique solution, $u \in B_{1}$, since we have supposed $T z=0$ only if $z=0$. Now the question of stability is this: If $T u_{n}=g_{n}$, and if the $g_{n}$ tend in some sense to $g$, do the $u_{n}$ tend to $u$ ?

The trivial case is that in which $T$ has a bounded inverse, $T^{-1}$. Then $u_{n} \rightarrow u$ if and only if $g_{n} \rightarrow g$, and $u_{n} \rightarrow u$ if and only if $g_{n} \rightarrow g$. The first assertion is obvious, and the second follows at once from the existence of bounded adjoint operators $T^{*}$ and $\left(T^{-1}\right)^{*}$. We note that if $B_{1}$ is finite-dimensional, then $T$ does have a bounded inverse, since we assume that $T$ is one-to-one, and that $T B_{1}$ is dense in $B_{2}$.

If $B_{1}$ is infinite-dimensional, $T$ may lack a bounded inverse and still satisfy our requirements. A simple example is given by the mapping of the space, $C$, of continuous functions $x(\tau)$ on $0 \leqq \tau \leqq 1$ into $L^{2}[0,1]$ by the transformation

$$
T x(\tau)=\int_{0}^{\tau} x(\sigma) d \sigma .
$$

Many examples are of the following form: Let $B_{1}$ and $B_{2}$ be identical Hilbert spaces. Let $T$ be a completely continuous, selfadjoint operator whose eigenvalues $\lambda_{n}$ are nonzero, and whose eigenvectors $v_{n}$ form a basis for $B_{1}$. Then a bounded inverse $T^{-1}$ cannot exist; for if $\left\|v_{n}\right\|=1$, then $v_{n} \rightarrow 0$ and hence $\lambda_{n} v_{n}=T v_{n} \rightarrow 0$.

3. Stability of Bounded Solutions. After proving the following general theorem, we will explain the need for reflexiveness, for boundedness, and for the use of weak, instead of strong, convergence.

THEOREM 1. Let $T$ be a bounded operator mapping a reflexive Banach space, $B_{1}$, into $a$ dense subset of a Banach space, $B_{2}$. Let $T z=0$ only if $z=0$. Let

$$
T u_{n}=g_{n} \quad(n=1,2, \cdots) .
$$

Assume that the $u_{n}$ are bounded: $\left\|u_{n}\right\| \leqq \beta$. Then $g_{n} \rightarrow g$ in $B_{2}$ if and only if there is $a$ point $u$ in $B_{1}$ for which $u_{n} \rightarrow u$, with $T u=g$.

Proof. We assert that $T^{*} B_{2}^{*}$ is dense in $B_{1}^{*}$. Otherwise, there would be an element $f_{0}$ in $B_{1}^{*}$ but not in the closure of $T^{*} B_{2}^{*}$. Using the Hahn-Banach theorem, we now construct a functional $\Phi(f)$ for all $f \in B_{1}^{*}$, with

$$
\Phi\left(f_{0}\right) \neq 0 \text {, but } \Phi(f)=0 \text { for } f \in T^{*} B_{2}^{*} .
$$

Since $\Phi \in B_{1}^{* *}=B_{1}$, there is a point $x_{0}$ in $B_{1}$ such that

$$
\Phi(f) \equiv\left(x_{0}, f\right) \text { for all } f \in B_{1}^{*} .
$$

If $f=T^{*} h \in T^{*} B_{2}^{*}$, then (2) and (3) imply

$$
0=\Phi(f)=\left(x_{0}, f\right)=\left(x_{0}, T^{*} h\right)=\left(T x_{0}, h\right) .
$$


Thus, $0=\left(T x_{0}, h\right)$ for all $h \in B_{2}^{*}$, which implies $T x_{0}=0$, and hence $x_{0}=0$. Now (3) implies $\Phi(f) \equiv 0$ for $f \in B_{1}^{*}$, which contradicts the first half of (2).

If $u_{n} \rightarrow u \in B_{1}$, then $T u_{n}=g_{n} \rightarrow T u=g$ because, for all $h \in B_{2}^{*}$,

$$
\left(g_{n}-T u, h\right)=\left(T\left(u_{n}-u\right), h\right)=\left(u_{n}-u, T^{*} h\right) \rightarrow 0 .
$$

Conversely, assuming $T u_{n}$ converges weakly, we can show that $u_{n}$ converges weakly. Let $T u_{n} \rightarrow g$. Let $f$ be given in $B_{1}^{*}$. Since $T^{*} B_{2}^{*}$ has been shown to be dense in $B_{1}^{*}$, given $\epsilon>0$ we can find a point $h \in B_{2}^{*}$ for which $\left\|f-T^{*} h\right\|<\epsilon$. Since

$$
\left(u_{n}-u_{m}, f\right)=\left(T\left(u_{n}-u_{m}\right), h\right)+\left(u_{n}-u_{m}, f-T^{*} h\right)
$$

we deduce, for all $n$ and $m$,

$$
\left|\left(u_{n}-u_{m}, f\right)\right| \leqq\left|\left(T u_{n}-T u_{m}, h\right)\right|+2 \beta \epsilon .
$$

Since $\left\{T u_{n}\right\}$ converges weakly, and since $\epsilon$ is arbitrarily small, we conclude that $\left(u_{n}-u_{m}, f\right) \rightarrow 0$ as $n$ and $m \rightarrow \infty$. Therefore, for every $f \in B_{1}^{*}$, there exists a limit

$$
L(f)=\lim _{n \rightarrow \infty}\left(u_{n}, f\right) .
$$

Now $L(f)$ is a bounded linear functional; indeed, $\|L\| \leqq \beta$, since all $\left\|u_{n}\right\| \leqq \beta$. Hence, $L \in B_{1}^{* *}=B_{1}$; and so $L$ has a representation

$$
L(f) \equiv(u, f) \quad \text { for all } f \in B_{1}^{*}
$$

where $u$ is some point in $B_{1}$, independent of $f$. From (7) and (8) we conclude that $u_{n} \rightarrow u$. Finally, $T u=g$ because, for all $h \in B_{2}^{*}$,

$$
(T u-g, h)=\lim _{n \rightarrow \infty}\left(T u_{n}-g, h\right)=0 .
$$

Having proved the theorem, we will explain the need for its hypotheses.

Univalence. If $T z=0$ for some $z \neq 0$, the theorem is false. For if $x_{n}=(-)^{n} z$, then $x_{n}$ does not tend to zero weakly (or strongly), while $g_{n}=T x_{n} \equiv 0$.

Reflexiveness. If we do not require $B_{1}$ to be reflexive, the theorem is false. For example, let $B_{1}$ be the space, $C$, of real-valued continuous functions $x=\psi(t)$ defined for $0 \leqq t \leqq 1$. Let $B_{2}=L^{2}[0,1]$. Let

$$
T x=\int_{0}^{t} \psi(\tau) d \tau \quad(0 \leqq t \leqq 1) .
$$

Note that $T z=0$ only if $z=0$. If $u_{n}=\psi_{n}(t)=\cos n t$, then

$$
g_{n}=T u_{n}=n^{-1} \sin n t \rightarrow 0 \quad \text { as } n \rightarrow \infty .
$$

Moreover, the $u_{n}$ are uniformly bounded:

$$
\left\|u_{n}\right\|=\max _{0 \leq t \leq 1}|\cos n t|=1 .
$$

But, if $\Phi$ is the functional which evaluates a function $\psi(t)$ for $t=\pi / 4$, then

$$
\Phi\left(u_{n}\right)=\cos (n \pi / 4)
$$

which diverges as $n \rightarrow \infty$. Hence, the sequence $\left\{u_{n}\right\}$ is not weakly convergent even though $g_{n}$ is strongly convergent. Incidentally, this example and Theorem 1 provide an independent proof that the Banach space $C$ is not reflexive; cf. [7, p. 214]. 
Weak convergence. If we do not use the notion of weak convergence, but use only strong convergence, the theorem reduces to the trivial case, in which $T$ has a bounded inverse:

Assertion. Let $T$ be a bounded operator mapping a Banach space $B_{1}$ into a dense subset of a Banach space $B_{2}$, with $T z=0$ only if $z=0$. (Here we do not need the assumption that $B_{1}$ is reflexive.) Let $T$ have the property that a sequence $\left\{x_{n}\right\}$ must converge strongly if it is bounded and if $\left\{T x_{n}\right\}$ converges strongly. Then $T B_{1}=B_{2}$, and $T$ has a bounded inverse, $T^{-1}$, mapping $B_{2}$ into $B_{1}$.

Proof. To show $T B_{1}=B_{2}$, let $y$ be any point in $B_{2}$. Since $T B_{1}$ is assumed to be dense in $B_{2}$, there is a sequence $\left\{x_{n}\right\}$ in $B_{1}$ such that $T x_{n} \rightarrow y$. If $\left\{x_{n}\right\}$ has a bounded subsequence, $\left\{x_{n}^{\prime}\right\}$, the hypothesis implies that $x_{n}^{\prime}$ has a strong limit, $x$, and hence $T x=y \in T B_{1}$. If $\left\{x_{n}\right\}$ has no bounded subsequence, then $\left\|x_{n}\right\| \rightarrow \infty$. If $x_{n} \neq 0$ for $n \geqq N$, define the points

$$
u_{2 n-1}=x_{n} /\left\|x_{n}\right\|, \quad u_{2 n}=0 \quad \text { for } n \geqq N .
$$

Since $T x_{n} \rightarrow y$ while $\left\|x_{n}\right\| \rightarrow \infty$, we have $T u_{n} \rightarrow 0$. Since $\left\|u_{n}\right\| \leqq 1$, the hypothesis implies the convergence of $\left\{u_{n}\right\}$. But $\left\{u_{n}\right\}$ diverges because $\left\|u_{2 n-1}-u_{2 n}\right\|=1$. Therefore, $\left\{x_{n}\right\}$ does have a bounded subsequence, and $T B_{1}=B_{2}$.

Hence, $T$ has an inverse, $T^{-1}$. If $T^{-1}$ were not bounded, there would be a sequence of unit vectors, $v_{n}$, for which $T v_{n} \rightarrow 0$. Then $T x_{n} \rightarrow 0$ if $x_{2 n-1}=v_{n}$ and $x_{2 n}=0$. The hypothesis now implies that $\left\{x_{n}\right\}$ converges, which is impossible because $\| x_{2 n-1}-$ $x_{n} \|=1$. This completes the proof of the assertion.

Boundedness. If we omit the assumption that $\left\{u_{n}\right\}$ is bounded, the theorem is false. For example, let $T$ be any bounded linear operator mapping a Hilbert space into itself, with unit eigenvectors $v_{1}, v_{2}, \cdots$ and associated nonzero eigenvalues $\lambda_{1}, \lambda_{2}, \cdots$ tending to zero as $n \rightarrow \infty$. Let $u_{n}=\left|\lambda_{n}\right|^{-1 / 2} v_{n}$. Then $T u_{n} \rightarrow 0$ while $\left\|u_{n}\right\| \rightarrow \infty$. Since a weakly convergent sequence must be bounded, $\left\{u_{n}\right\}$ is not weakly convergent.

4. An Example for the Heat Equation. To show how the theorem can be used, we will consider the stability of the flux of heat depending upon a temperature. Consider the conduction of heat in a semi-infinite, homogeneous rod $0 \leqq x<\infty$ in an interval of time $0 \leqq t \leqq t_{1}$. If $\varphi(x, t)$ is the temperature, assume that it obeys the equation

$$
\frac{\partial \varphi}{\partial t}=\frac{\partial^{2} \varphi}{\partial x^{2}} \quad\left(0 \leqq x<\infty, 0 \leqq t \leqq t_{1}\right)
$$

Let the temperature at $x=0$ be given:

$$
\varphi(0, t)=g(t) \quad\left(0 \leqq t \leqq t_{1}\right)
$$

and let the unknown be the flux of heat into the end at $x=0$ :

$$
-\frac{\partial}{\partial x} \varphi(0, t)=u(t) \quad\left(0 \leqq t \leqq t_{1}\right) .
$$

We assume the initial and boundary conditions

$$
\varphi(x, 0) \equiv 0 \text { and } \varphi(+\infty, t) \equiv 0 .
$$


Define the Laplace transform

$$
\Phi(x, s)=\int_{0}^{\infty} e^{-s t} \varphi(x, t) d t \quad(\operatorname{Re} s>0)
$$

and let the boundary temperature, $g(t)$, and flux, $u(t)$, have the transforms $G(s)$ and $U(s)$. Then the preceding equations yield

$$
s \Phi(x, s)=\frac{\partial^{2}}{\partial x^{2}} \Phi(x, s)
$$

and

(5) $\quad \Phi(x, s)=G(s) \exp (-x \sqrt{ } s), \quad U(s)=-\frac{\partial}{\partial x} \Phi(0, s)=\sqrt{ } s G(s)$.

Since $s^{-1 / 2}$ is the Laplace transform of $(t / \pi)^{1 / 2}$, the convolution theorem yields

$$
\frac{1}{\sqrt{ } \pi} \int_{0}^{t}(t-\tau)^{-1 / 2} u(\tau) d \tau=g(t) \quad\left(0 \leqq t \leqq t_{1}\right) .
$$

This is an equation, $T u=g$, for which we will discuss the stability of the solution, $u$. We will not use the explicit solution of the Abel integral equation (6), which is

$$
u(t)=\frac{d}{d t} \frac{1}{\sqrt{ } \pi} \int_{0}^{t}(t-\tau)^{-1 / 2} g(\tau) d \tau
$$

For the sake of definiteness, consider the example

$$
g_{0}(t) \equiv 1, \quad u_{0}(t)=(\pi t)^{-1 / 2} \quad\left(0 \leqq t \leqq t_{1}\right) .
$$

The function $u(t)$ is not square integrable, but it does lie in the reflexive Banach space $B_{1}=L^{D}\left[0, t_{1}\right]$ if $1<p<2$. We will now show that $T$ is a bounded linear operator mapping $L^{p}\left[0, t_{1}\right]$ into $L^{2}\left[0, t_{1}\right]$.

Given the equation (6), with $u \in L^{p}$, we must show that

$$
\int_{0}^{t_{1}} g^{2}(t) d t<\infty
$$

From (6) we find

$$
\int_{0}^{t_{1}} g^{2}(t) d t=\int_{0}^{t_{1}} \int_{0}^{t_{1}} H(\sigma, \tau) u(\sigma) u(\tau) d \sigma d \tau
$$

where

$$
H(\sigma, \tau)=\frac{1}{\pi} \int_{\max (\sigma, \tau)}^{t_{1}}[(t-\tau)(t-\sigma)]^{-1 / 2} d t
$$

or

$$
H(\sigma, \tau)=\frac{1}{\pi} \log \frac{2 t_{1}-\sigma-\tau+2\left[\left(t_{1}-\sigma\right)\left(t_{1}-\tau\right)\right]^{1 / 2}}{|\tau-\sigma|}
$$

Since

$$
0 \leqq H(\sigma, \tau) \leqq \frac{1}{\pi} \log \frac{4 t_{1}}{|\tau-\sigma|}
$$


we have, for all $q>0$,

$$
\eta_{a} \equiv \int_{0}^{t_{2}} \int_{0}^{t_{1}}\{H(\sigma, \tau)\}^{a} d \sigma d \tau<\infty .
$$

If $q=\left(1-p^{-1}\right)^{-1}$, Hölder's inequality applied to (10) yields

$$
\int_{0}^{t_{1}} g^{2}(t) d t \leqq\left(\eta_{Q}\right)^{1 / a}\left(\int_{0}^{t_{1}}|u(t)|^{p} d t\right)^{2 / p}<\infty .
$$

This completes the proof that $T$ is bounded.

If $g(t)=0$, then the boundary-value problem (1), (2), (4) has the unique solution $\varphi(x, t) \equiv 0$. Then $u(t)=-\varphi_{x}(0, t)=0$. Therefore, $T u=0$ only if $u=0$.

Theorem 1 now yields the following result: Let $u_{n}(t)$ satisfy

$$
\int_{0}^{t_{1}}\left|u_{n}(t)\right|^{p} d t \leqq \beta^{p} \quad(n=1,2, \cdots)
$$

where $\beta$ is a finite bound independent of $n$. Let $\left\{g_{n}(t)\right\}$ converge weakly in $L^{2}$ to $g_{0}(t)$, i.e., let

$$
\int_{0}^{t_{2}}\left[g_{n}(t)-g_{0}(t)\right] w(t) d t \rightarrow 0 \quad \text { as } n \rightarrow \infty
$$

for all $w(t) \in L^{2}$. Then $\left\{u_{n}(t)\right\}$ converges weakly in $L^{p}$ to $u_{0}(t)$, i.e.,

$$
\int_{0}^{t_{1}}\left[u_{n}(t)-u_{0}(t)\right] \psi(t) d t \rightarrow 0 \quad \text { as } n \rightarrow \infty
$$

for all $\psi(t) \in L^{a}$, where $q=\left(1-p^{-1}\right)^{-1}$.

It is noteworthy that $T$ does not have a bounded inverse, $T^{-1}$, mapping $L^{2}$ into $L^{p}$. To see this, let $u_{n}(t)=\cos n t$. Then

$$
g_{n}(t)=T u_{n}(t)=\frac{1}{\sqrt{ } \pi} \int_{0}^{t}(t-\tau)^{-1 / 2} \cos n \tau d \tau
$$

which is $O\left(n^{-1 / 2}\right)$ as $n \rightarrow \infty$ uniformly in $t$ for $0 \leqq t \leqq t_{1}$. Therefore, $\left\|g_{n}\right\| \rightarrow 0$ as $n \rightarrow \infty$. But, since $p<2$,

$$
\left\|u_{n}\right\|^{p}=\int_{0}^{t_{1}}|\cos n t|^{p} d t \geqq \int_{0}^{t_{1}}(\cos n t)^{2} d t \rightarrow \frac{1}{2} t_{1}>0 \quad \text { as } n \rightarrow \infty .
$$

Thus, $\left\|g_{n}\right\| \rightarrow 0$ while $\left\|T g_{n}\right\|$ is bounded away from zero.

The practical use of the weak convergence (17) is illustrated as follows. Usually one is not so much interested in the instantaneous flux, $u(t)$, as in the total flux

$$
F=\int_{t_{0}}^{t_{0}+\Delta t} u(t) d t
$$

during some nonzero time interval, $t_{0} \leqq t \leqq t_{0}+\Delta t$. Let $\psi(t) \equiv 1$ in this interval and $\psi(t) \equiv 0$ elsewhere. Then

$$
F_{n}=\int_{t_{0}}^{t_{0}+\Delta t} u_{n}(t) d t=\int_{0}^{t_{1}} u_{n}(t) \psi(t) d t .
$$

Since $\psi(t) \in L^{q}$, the weak convergence (17) implies that $F_{n} \rightarrow F_{0}$ as the data $g_{n}(t)$ 
tends to $g_{0}(t)$ provided that the instantaneous fluxes $u_{n}(t)$ are uniformly bounded in $L^{p}$, which is the assumption (15).

5. Application to Elasticity. Consider the biharmonic equation

$$
\Delta^{2} \varphi=0
$$

in two dimensions. If $\varphi$ and $\partial \varphi / \partial n$ are prescribed on a simple closed curve, $C_{1}$, and if $P$ is a point in the domain, $D_{1}$, enclosed by $C_{1}$, then there is a formula (cf. Garabedian $[8$, p. 266])

$$
\varphi(P)=\int_{C_{1}}\left[A(P, Q) \varphi(Q)+B(P, Q) \frac{\partial \varphi}{\partial n}(Q)\right] d s
$$

where $A(P, Q)$ and $B(P, Q)$ are analytic functions of the Cartesian coordinates of $P$ in the open set $D_{1}$.

Let $C_{2}$ be a simple closed curve in $D_{1}$, as in the figure.

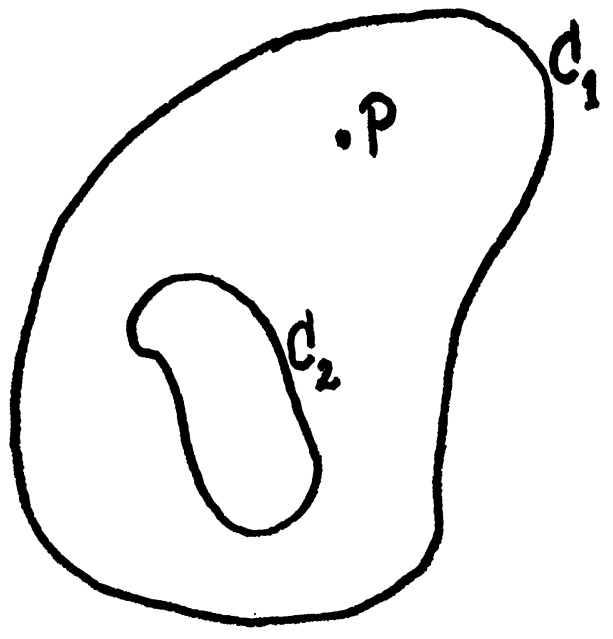

FIGURE 1

Suppose that $\varphi$ and $\partial \varphi / \partial n$ are prescribed on the inner curve, $C_{2}$. We now inquire about the stability of the solution, $\varphi$, at a point, $P$, outside $C_{2}$. We will consider only functions $\varphi$ which solve the biharmonic equation in the larger domain, $D_{1}$, and which satisfy on the outer curve, $C_{1}$, an inequality

$$
\int_{C_{1}}\left\{[\varphi(Q)]^{2}+[\partial \varphi(Q) / \partial n]^{2}\right\} d s \leqq \beta^{2}
$$

where $\beta$ is a finite number independent of $\varphi$

Let $g$ be defined as the pair of real-valued functions $\varphi\left(P_{2}\right), \partial \varphi\left(P_{2}\right) / \partial n$ for $P_{2}$ on $C_{2}$. Let

$$
\|g\|^{2}=\int_{C_{3}}\left\{\left[\varphi\left(P_{2}\right)\right]^{2}+\left[\partial \varphi\left(P_{2}\right) / \partial n\right]^{2}\right\} d s .
$$

Similarly, let $u$ be the pair of functions $\varphi(Q), \partial \varphi(Q) / \partial n$; and define $\|u\|^{2}$ by the left- 
hand side of (3). By using Green's formula (2) and its first derivatives, with $P$ replaced by $P_{2}$ on $C_{2}$, one obtains an operator, $T$, which relates $u$ to $g$ :

$$
T u=g .
$$

Let $B_{1}$ be the Hilbert space of pairs of functions $\varphi(Q), \psi(Q)$ which are squareintegrable on $C_{1}$. Similarly, define $B_{2}$ on $C_{2}$. Then Green's formula shows that $T$ is a bounded linear operator mapping $B_{1}$ into $B_{2}$. The application of $T$ to polynomials in two variables shows that $T B_{1}$ is dense in $B_{2}$. Moreover, $T z=0$ only if $z=0$; for if $\varphi$ and $\partial \varphi / \partial n$ vanish on $C_{2}$, then $\varphi \equiv 0$ in $D_{2}$, and the analyticity of $\varphi$ implies that $\varphi \equiv 0$ in the larger domain, $D_{1}$, so that $\varphi$ and $\partial \varphi / \partial n$ also vanish on $C_{1}$.

Let $g_{1}, g_{2}, \cdots$ be a sequence of data converging weakly in $B_{2}$. This will be true if the corresponding functions $\varphi_{1}\left(P_{2}\right), \varphi_{2}\left(P_{2}\right), \cdots$ and their normal derivatives on $C_{2}$ converge uniformly for $P_{2}$ on $C_{2}$. Less is required; since the $\left\|g_{k}\right\|$ have a uniform bound, it suffices to assume that the limit

$$
\lim _{k \rightarrow \infty} \int_{C_{2}}\left[a\left(P_{2}\right) \varphi_{k}\left(P_{2}\right)+b\left(P_{2}\right) \frac{\partial \varphi_{k}\left(P_{2}\right)}{\partial n}\right] d s
$$

exists for every pair of functions, $a\left(P_{2}\right)$ and $b\left(P_{2}\right)$, chosen from a dense subset of $L^{2}\left(C_{2}\right)$.

Theorem 1 now implies the weak convergence of $u_{k}$ in $B_{1}$, i.e., the weak convergence of the pair of functions $\varphi, \partial \varphi / \partial n$ on the Cartesian product $L^{2}\left(C_{1}\right) \times L^{2}\left(C_{1}\right)$.

If, as in the figure, $P$ is a fixed point between $C_{2}$ and $C_{1}$, Green's formula (2) represents the number $\varphi(P)$ as an inner product in $B_{1}$; and differentiation of Green's formula represents all derivatives of $\varphi$ as inner products. Hence, the weak convergence of $u_{k}$ implies that $\varphi_{k}$ and all of its derivatives converge pointwise as $k \rightarrow \infty$, i.e., as the data $\varphi, \partial \varphi / \partial n$ converge on the inner curve, $C_{2}$. Of course, if $P$ had been chosen inside $C_{2}$, the convergence would have been an immediate consequence of Green's formula for the inner region, $D_{2}$.

6. Stability of Harmonic Continuation. If, in the preceding section, we had considered Laplace's equation, $\Delta \varphi=0$, instead of the biharmonic equation, using boundary values only for $\varphi$ and not for $\partial \varphi / \partial n$, we would have obtained a classical result: If $\Delta \varphi_{k}=0$ in the larger domain, $D_{1}$, and if the $\varphi_{k}$ are uniformly bounded in $D_{1}$, and if the $\varphi_{k}$ converge on the inner curve, $C_{2}$, then $\varphi_{k}$ and all its derivatives converge at points $P$ between $C_{2}$ and $C_{1}$, as well as at points inside $C_{2}$.

If $E$ is an elliptic operator of order $2 k$, these results generalize immediately for the boundary-value problem with prescribed values for $\varphi$ and its normal derivatives of orders less than $k$.

7. The Final-Value Problem. Richtmyer's book [6] discusses the initial-value problem

$$
d v(t) / d t=A v(t) \quad\left(0 \leqq t \leqq t_{1}\right), \quad v(0)=u,
$$

where, for each $t, v(t)$ is an element in a Banach space, $B$. The operator $A$ is a linear, bounded or unbounded operator whose domain is dense in $B$, and whose range lies in $B$. There is a solution-operator, $E(t)$, which is supposed to have a bounded extension to all of $B$. Thus, if $v\left(t_{1}\right)=g$ and $E\left(t_{1}\right)=T$, the initial-value problem, to determine 
$g$ from $u$, is well-posed; it has the solution

$$
T u=g .
$$

The form (1) has been used to discuss wave motion, heat transfer, neutron transport, and elastic vibration.

By the final-value problem, we mean that of determining $u=v(0)$ from $g=v\left(t_{1}\right)$ in a well-posed initial-value problem (1). The backward heat equation is of this type. It is an ill-posed problem, since the solutions are unstable. However, the backward wave equation is well-posed. Under certain general conditions, we will show that uniformly bounded solutions of the final-value problem are stable.

We will give a commonly-met condition on $A$ which ensures that $T z=0$ only if $z=0$. Let there be a set of points $f_{1}, f_{2}, \cdots$ in $B^{*}$ such that, for all $w$ in the domain of $A$

$$
\left(A w, f_{n}\right)=\alpha_{n}\left(w, f_{n}\right) \quad(n=1,2, \cdots)
$$

where $\alpha_{1}, \alpha_{2}, \cdots$ are certain scalars. Thus, the points $f_{n}$ are eigenvectors in the domain of $A^{*}$. Assume that the $\left\{f_{n}\right\}$ are complete in $B^{*}$, in the sense that

$$
\left(z, f_{n}\right)=0 \text { for all } n \text { only if } z=0 \text {. }
$$

For example, if $B=L^{2}[0,1]$, and if $A \psi(x)=\psi^{\prime \prime}(x)$, the second derivative of $w=$ $\psi(x)$, and if the domain of $A$ consists of the functions $\psi(x)$ with two continuous derivatives such that $\psi(0)=\psi(\pi)=0$, then $B=B^{*}, A=A^{*}$, and we may choose

$$
f_{n}=\sin n \pi x, \quad \alpha_{n}=-n^{2} \pi^{2} .
$$

Then (4) states that the set $\{\sin n \pi x\}$ is complete in $L^{2}[0,1]$.

If $A$ satisfies the preceding condition, then $T z=0$ only if $z=0$. For let $v(t)$ satisfy the initial-value problem $(1)$ with $v(0)=z$. Now, for each $f_{n}$,

$$
\frac{d}{d t}\left(v(t), f_{n}\right)=\left(A v(t), f_{n}\right)=\alpha_{n}\left(v(t), f_{n}\right) \quad\left(0 \leqq t \leqq t_{1}\right)
$$

by the assumption (3). Therefore, for $0 \leqq t \leqq t_{1}$ and for $n=1,2, \cdots$,

$$
\left(v(t), f_{n}\right)=\left(z, f_{n}\right) \exp \left(\alpha_{n} t\right) .
$$

When $t=t_{1}$, we have $v\left(t_{1}\right)=T z=0$, and hence (6) implies

$$
0=\left(z, f_{n}\right) \quad(n=1,2, \cdots) .
$$

Now (4) implies $z=0$.

Applied to the preceding example, this argument implies the well-known uniqueness of solutions to the final-value problem for the heat equation $\varphi_{t}=\varphi_{x x}$ with boundary condition $\varphi=0$ for $x=0, \pi$. If, more generally,

$$
A \psi(x)=\left(p(x) \psi^{\prime}(x)\right)^{\prime}+q(x) \psi(x)
$$

with $p(x)>0$, and with $p(x), p^{\prime}(x)$, and $q(x)$ continuous, the completeness of eigenfunctions for Sturm-Liouville operators implies the uniqueness of solutions to the final-value problem for

$$
\frac{\partial \varphi}{\partial t}(x, t)=\frac{\partial}{\partial x}\left(p(x) \frac{\partial \varphi}{\partial x}\right)+q(x) \varphi
$$


To ensure that $T B$ is dense in $B$, it suffices to assume that eigenvectors $w_{1}, w_{2}, \ldots$ whose finite linear combinations are dense in $B$. For then, if $A w_{v}=\beta_{v} w_{v}$, we have

$$
T \sum_{\nu=1}^{n} \gamma_{\nu} w_{\nu}=\sum_{\nu=1}^{n} \gamma_{\nu} \exp \left(\beta_{\nu} t_{1}\right) w_{\nu}
$$

and the linear combinations (9) are dense in $B$.

In summary, we have proved the following result:

LeMma. If $A$ has eigenvectors $w_{n}$ whose finite linear combinations are dense in $B$, and if $A^{*}$ has eigenvectors $f_{n}$ which are complete in $B^{*}$, then the well-posed initial-value problem (1) has a solution-operator, $T$, which maps $B$ into a dense subset of $B$, with $T z=0$ only if $z=0$.

Theorem 1 now yields the following criterion for the stability of solutions of the terminal-value problem:

THEOREM 2. Let the well-posed initial-value problem (1) be defined for a reflexive Banach space, $B$. Let $T u_{n}=g_{n}(n=1,2, \cdots)$ where the initial values $u_{n}$ are uniformly bounded: $\left\|u_{n}\right\| \leqq \beta$. Let A satisfy the conditions of the lemma. Then the weak convergence of the final values, $g_{n}$, implies the weak convergence of the initial values, $u_{n}$.

The weak convergence of the initial values ensures the strong convergence of the vectors $v_{v}(t)$ for $0<t<t_{1}$ if the solution operator $E(t)$ is completely continuous for $t>0$. This is true, for instance, for the heat equation (8) involving a general SturmLiouville operator, $A$. If a complete set of eigenvectors is the set of orthonormal functions $w_{n}=\varphi_{n}(x)$, then the eigenvalues $\beta_{n}$ tend to $+\infty$ like a constant multiple of $n^{2}$, and

$$
E(t) \sum_{1}^{\infty} \gamma_{n} \varphi_{n}(x)=\sum_{1}^{\infty} \gamma_{n} \exp \left(-\beta_{n} t\right) \varphi_{n}(x) .
$$

Here we assume $\sum \gamma_{n}^{2}<\infty$, and the convergence of the infinite series $\sum \gamma_{n} \varphi_{n}(x)$ is understood in the mean-square sense. If the initial values $u_{1}, u_{2}, \cdots$ have series representations

$$
u_{k}=\sum_{n=1}^{\infty} c_{k n} \varphi_{n}(x) \quad(k=1,2, \cdots)
$$

and if

$$
u=\sum_{n=1}^{\infty} c_{n} \varphi_{n}(x)
$$

in $L^{2}$, then weak convergence $u_{k} \rightarrow u$ is equivalent to the conditions

$$
\begin{array}{ll}
\sum_{n=1}^{\infty} c_{k n}^{2} \leqq c^{2} & \text { independent of } \mathrm{k}, \\
\lim _{k \rightarrow \infty} c_{k n}=c_{n} & \text { for each fixed } n .
\end{array}
$$

Applying (10), we find, for $t>0$,

$$
v_{k}(t)=E(t) u_{k}=\sum_{n=1}^{\infty} \gamma_{k n} \exp \left(-\beta_{n} t_{0}\right) \varphi_{n}(x) .
$$


Since $\beta_{n} \rightarrow+\infty$ as $n \rightarrow \infty$, the conditions of weak convergence, (12) and (13), imply the strong convergence

$$
\lim _{k \rightarrow \infty}\left\|E(t) u_{k}-E(t) u\right\|=0 \quad \text { if } t>0 .
$$

This follows at once from the inequality

$$
\begin{aligned}
\| \sum_{n=1}^{\infty}\left(\gamma_{k n}-\right. & \left.\gamma_{n}\right) \exp \left(-\beta_{n} t\right) \varphi_{n}(x) \|^{2} \\
& \leqq \sum_{n=1}^{N-1}\left(\gamma_{k n}-\gamma_{n}\right)^{2} \exp \left(-2 \beta_{n} t\right)+2 \exp \left(-2 \beta_{N} t\right)\left(c^{2}+\sum \gamma_{n}^{2}\right)
\end{aligned}
$$

which holds for each $N \geqq 1$.

In this example, we are even able to deduce the pointwise convergence

$$
\varphi_{k}\left(x_{0}, t\right) \rightarrow \varphi\left(x_{0}, t\right) \quad \text { as } k \rightarrow \infty \text { if } t>0 .
$$

For fixed $x_{0}$ and $t>0$, the real number $\varphi_{k}\left(x_{0}, t\right)$ is the inner product of the vector $u_{k}=\varphi_{k}(x, 0)$ with the vector in $L^{2}$ whose Fourier series is

$$
s=\sum_{n=1}^{\infty}\left[\varphi_{n}\left(x_{0}\right) \exp \left(-\beta_{n} t\right)\right] \varphi_{n}(x)
$$

where, since $t_{0}>0$,

$$
\|s\|^{2}=\sum_{n=1}^{\infty}\left[\varphi_{n}\left(x_{0}\right) \exp \left(-\beta_{n} t\right)\right]^{2}<\infty .
$$

(Here we have used the result $\sum \beta_{v}^{-1} \varphi_{n}^{2}\left(x_{0}\right)<\infty$ from Sturm-Liouville theory.) Now the weak convergence $u_{k} \rightarrow u$ yields

$$
\left(u_{k}, s\right) \rightarrow(u, s) \quad \text { as } k \rightarrow \infty
$$

which is the asserted pointwise convergence (17).

Acknowledgment. I wish to thank Professors David Boyd and Stanley A. Weiss for their helpful comments.

Applied Mathematics Department California Institute of Technology Pasadena, California 91109

1. J. Hadamard, Lectures on Cauchy's Problem in Linear Partial Differential Equations, Yale Univ. Press, New Haven, Conn., 1923.

2. F. JOHN, "Numerical solution of the equation of heat conduction for preceding times," Ann. Mat. Pura Appl. (4), v. 40, 1955, pp. 129-142. MR 19, 323.

3. F. JoHN, Numerical Solution of Problems which are not Well Posed in the Sense of Hadamard, Proc. Sympos. Numerical Treatment of Partial Differential Equations with Real Characteristics (Rome, 1959), Libreria Eredi Virgilio Veschi, Rome, 1959, pp. 103-116. MR 21 \#6704.

4. F. JoHN, "Continuous dependence on data for solutions of partial differential equations with a prescribed bound," Comm. Pure Appl. Math., v. 13, 1960, pp. 551-585. MR 24 \#A317. 
5. L. E. Payne, On Some Nonwell Posed Problems for Partial Differential Equations, Proc. Adv. Sympos. Numerical Solutions of Nonlinear Differential Equations (Madison, Wis., 1966), Wiley, New York, 1966, pp. 239-263. MR 35 \#4606.

6. R. D. RICHTMYER, Difference Methods for Initial-Value Problems, Interscience Tracts in Pure and Appl. Math., Tract 4, Interscience, New York, 1957. MR 20 \#438.

7. F. RIESz \& B. Sz.-NAGY, Lecons d'Analyse Fonctionnelle, Akad. Kiadó, Budapest, 1953; English transl., Ungar, New York, 1955. MR 15, 132; MR 17, 175. \#5247.

8. P. R. Garabedian, Partial Differential Equations, Wiley, New York, 1964. MR 28

9. J. R. Gannon, Some Numerical Results for the Solution of the Heat Equation Backwards in Time, Proc. Adv. Sympos. Numerical Solutions of Nonlinear Differential Equations (Madison, Wis., 1966), Wiley, New York, 1966, pp. 21-54. MR 34 \#7037.

10. J. Douglas, JR., Approximate Continuation of Harmonic and Parabolic Functions, Proc. Sympos. Numerical Solution of Partial Differential Equations (Univ. of Maryland, 1965), Academic Press, New York, 1966, pp. 353-364. MR 34 \#2206.

11. R. SAYLOR, "Numerical elliptic continuation," SIAM J. Numer. Anal., v. 4, 1967, pp. 575-581. MR 36 \#5513. 